\title{
STATES ON QUANTUM LOGICS AND THEIR CONNECTION WITH A THEOREM OF ALEXANDROFF
}

\author{
O. R. BÉAVER AND T. A. COOK
}

\begin{abstract}
We generalize the notion of regularity of measures to quantum logics and then prove that each regular finitely additive state on a quantum logic is countably additive. Examples are given from measure theory and quantum mechanics.
\end{abstract}

In this note, we establish a generalization of the well-known theorem of Alexandroff: Each bounded, regular and finitely additive measure on a compact Hausdorff space is countably additive [2, p. 138].

Let $(\mathcal{L}, \leqslant)$ be a partially ordered set with at least two elements, a largest element $e$ and a smallest element 0 . Let $\mathcal{L}$ be equipped with an orthocomplementation '. Specifically, if $p \in \mathcal{L}$ then $p^{\prime} \in \mathcal{L}$ and the following are satisfied: (i) $\left(p^{\prime}\right)^{\prime}=p$ for all $p \in \mathcal{L}$; (ii) $p \leqslant q$ in $\mathcal{L}$ implies $q^{\prime} \leqslant p^{\prime}$; (iii) $p \vee p^{\prime}=e$ and $p \wedge p^{\prime}=0$ for all $p \in \mathcal{L}$. We say that $p, q$ are orthogonal, denoted $p \perp q$, if $p \leqslant q^{\prime}$. We will assume that if $\left\{p_{1}, p_{2}, \ldots, p_{n}\right\}$ is a finite

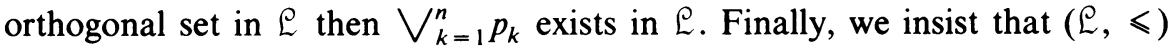
satisfies the orthomodular identity: $p \leqslant q$ in $\mathcal{L}$ implies $q=p \vee\left(q \wedge p^{\prime}\right)$. A set $\mathcal{L}$ satisfying all the conditions above will be called a quantum logic and its elements propositions. $(\mathcal{L}, \leqslant)$ is called $\sigma$-orthocomplete if for each countable orthogonal sequence $\left\{p_{1}, p_{2}, \ldots\right\}$ in $\mathcal{L}$ there exists $p \in \mathcal{L}$ such that $p=$ $\bigvee_{k=1}^{\infty} p_{k}$. We note here that a quantum logic need not be a lattice.

A state $\omega: \mathcal{L} \rightarrow[0,1]$ is a function with the properties: (i) $\omega(0)=0$ and $\omega(e)=1$; (ii) if $\left\{p_{1}, p_{2}, \ldots, p_{n}\right\}$ is a finite orthogonal set in $\mathcal{L}$ with $p=$ $\bigvee_{k=1}^{n} p_{k}$, then $\omega(p)=\sum_{k=1}^{n} \omega\left(p_{k}\right)$. A state $\omega$ is called countably additive if $\left\{p_{1}, p_{2}, \ldots\right\}$ is an orthogonal sequence with $p=\bigvee_{k=1}^{\infty} p_{k}$ in $\mathcal{L}$ then $\omega(p)=$ $\sum_{k=1}^{\infty} \omega\left(p_{k}\right)$. Let $\mathscr{P} \subseteq \mathcal{L}$; a state $\omega$ is called $\mathscr{P}$-regular if for each $\varepsilon>0$ and each $q \in \mathcal{L}$ there exists $p \in \mathcal{P}$ with $p \leqslant q$ and $\omega\left(q \wedge p^{\prime}\right)<\varepsilon$. If $\omega$ is $\mathcal{P}$-regular then for each $\varepsilon>0$ and $q \in \mathcal{L}$ there exists $p^{\prime} \in \mathcal{L}$ for some $p \in \mathcal{P}$ such that $p^{\prime} \geqslant q$ and $\omega\left(p^{\prime} \wedge q^{\prime}\right)<\varepsilon$. (There exists $p \in \mathcal{P}$ such that $p \leqslant q^{\prime}$ and $\omega\left(p^{\prime} \wedge q^{\prime}\right)<\varepsilon$. $)$

Let $\mathscr{P}^{\prime}=\left\{p^{\prime}: p \in \mathscr{P}\right\}$. An element $p \in \mathscr{P}$ is called finitely-coverable if $\left\{p_{1}^{\prime}, p^{\prime}{ }_{2}, \ldots\right\} \subseteq \mathscr{P}^{\prime}, \bigvee_{k=1}^{\infty} p^{\prime}{ }_{k}$ exists in $\mathcal{L}$ and $p \leqslant \bigvee_{k=1}^{\infty} p^{\prime}{ }_{k}$ implies for some $n$ that $\bigvee_{k=1}^{n} p^{\prime}{ }_{k}$ exists in $\mathcal{L}$ and $p \leqslant \bigvee_{k=1}^{n} p^{\prime}{ }_{k}$. $\mathcal{P}$ is called finitelycoverable if each element of $\mathcal{P}$ is finitely-coverable. We now state the

Received by the editors January 20, 1977 and, in revised form, April 25, 1977.

AMS (MOS) subject classifications (1970). Primary 28A60, 81A12, 06A25, 46G99.

Key words and phrases. Quantum logics, states, regular measures. 
generalized form of Alexandroff's theorem.

THEOREM. Suppose $\mathcal{L}$ has the following properties: $\mathcal{L}$ is a $\sigma$-orthocomplete quantum logic; $\mathcal{P} \subseteq \mathcal{L}$ is finitely-coverable; $\mathcal{P}^{\prime}$ contains the join of any sequence in $\mathcal{P}^{\prime}$ and $\omega$ is a $\mathcal{P}$-regular state on $\mathcal{L}$. Then $\omega$ is countably additive on e.

Proof. Let $\left\{q_{1}, q_{2}, \ldots\right\}$ be an orthogonal sequence in $\mathcal{L}$ with $q=\bigvee_{k=1}^{\infty} q_{k}$ in $\mathcal{L}$. Then $q \geqslant \bigvee_{k=1}^{n} q_{k}$ for all $n$ and $1 \geqslant \omega(q) \geqslant \sum_{k=1}^{n} \omega\left(q_{k}\right)$. Hence, $\omega(q) \geqslant$ $\sum_{k=1}^{\infty} \omega\left(q_{k}\right)$. Let $\varepsilon>0$ be given; then there exists $\left\{p_{1}, p_{2}, \ldots\right\} \subseteq \mathcal{P}$ with $p_{k}^{\prime} \geqslant q_{k}$ and $\omega\left(q_{k}^{\prime} \wedge p^{\prime}{ }_{k}\right)<\varepsilon / 2^{k}$. Also, there exists $p \in \mathscr{P}$ with $p \leqslant q$ and $\omega\left(q \wedge p^{\prime}\right)<\varepsilon$. Since $p$ is finitely-coverable, there exists $m$ such that $p \leqslant$ $\bigvee_{k=1}^{m} p_{k}^{\prime}$. From the orthomodularity of $\mathcal{E}$ and finite additivity of $\omega, \omega\left(p_{k}^{\prime}\right)=$ $\omega\left(q_{k}\right)+\omega\left(q^{\prime}{ }_{k} \wedge p^{\prime}{ }_{k}\right)$, and this implies $\omega\left(p^{\prime}{ }_{k}\right)-\varepsilon / 2^{k} \leqslant \omega\left(q_{k}\right)$. Hence, $\sum_{k=1}^{\infty} \omega\left(q_{k}\right) \geqslant \sum_{k=1}^{\infty} \omega\left(p^{\prime}{ }_{k}\right)-\varepsilon \geqslant \sum_{k=1}^{m} \omega\left(p^{\prime}{ }_{k}\right)-\varepsilon \geqslant \omega(p)-\varepsilon \geqslant \omega(q)-2 \varepsilon$. Therefore, $\omega(q)=\sum_{k=1}^{\infty} \omega\left(q_{k}\right)$.

We now give two examples. First, let $X$ be a compact Hausdorff space; identify $\mathcal{L}$ with the $\sigma$-algebra of Borel sets of $X$ and let $\mathscr{P}$ be the closed sets of $X$. Since $\mathscr{P}^{\prime}$ consists of the open sets, $\mathcal{P}^{\prime}$ is closed under countable joins and $\mathscr{P}$ is finitely-coverable. If $\omega$ is a finitely additive, regular, probability measure on $\mathcal{L}$ then $\omega$ is countably additive.

Second, let $\mathcal{L}$ be the $\sigma$-complete orthomodular lattice of projections on a separable Hilbert space and let $\mathscr{P}$ be the set of all projections each of which has finite dimensional range, see [1] or [3]. Each element $p \in \mathcal{L}$ (not only those in $\mathcal{P}$ ) are finitely-coverable. To see this let $p \in \mathcal{L}$ and let $\left\{p_{1}{ }_{1}, p^{\prime}{ }_{2}, \ldots\right\} \infty$ a sequence in $\mathcal{P}^{\prime}$ such that $p \leqslant \bigvee_{k=1}^{\infty} p^{\prime}{ }_{k}$. Each $p_{k}^{\prime}$ has as its range a subspace with finite co-dimension. If the integer $n_{k}, k=1,2, \ldots$ is the co-dimension of the range of $p^{\prime}{ }_{1} \vee \cdots \vee p^{\prime}{ }_{k}$ then $n_{1} \geqslant n_{2} \geqslant \cdots \geqslant 0$. Thus, for some $m, n_{m}=n_{m+1}=\cdots$ and $p \leqslant \bigvee_{k=1}^{m} p_{k}^{\prime}$. If $\omega$ is a $\mathscr{P}$-regular state then $\omega$ is countably additive. The countably additive states in this case have been identified by Gleason, see, e.g., $[3$, p. 132] as the positive trace class operators of unit trace. The fact that each such state is $\mathscr{P}$-regular follows from the spectral theorem. Let $\omega=\Sigma_{n} \lambda_{n} e_{n} \otimes \bar{e}_{n}$ and $p=\Sigma_{k} f_{k} \otimes \bar{f}_{k}$ where $\left(e_{n}\right)$ and $\left(f_{k}\right)$ are orthonormal sequences and $\lambda_{n}>0$ with $\Sigma_{n} \lambda_{n}=1$, see [1]. Then $\omega(p)=\operatorname{tr}(\omega \circ p)=\sum_{n, k} \lambda_{n}\left|\left(e_{n}, f_{k}\right)\right|^{2} \leqslant 1$. For $\varepsilon>0$ there exists $m$ sufficiently large for which $q=\sum_{k=1}^{m} f_{k} \otimes \bar{f}_{k}$ has the property:

$$
\omega\left(p \wedge q^{\prime}\right)=\omega(p)-\omega(q)=\sum_{k>m} \lambda_{n}\left|\left(e_{n}, f_{k}\right)\right|^{2}<\varepsilon \text {. }
$$

\section{REFERENCES}

1. T. A. Cook, The geometry of generalized quantum logics, Internat. J. Theoret. Phys. (to appear).

2. N. Dunford and J. Schwartz, Linear operators. I, Interscience, New York, 1957.

3. J. M. Jauch, Foundations of quantum mechanics, Addison-Wesley, Reading, Mass., 1968.

Department of Mathematics and Statistics, University of Massachusetts, Amherst, MASSACHUSETTS 01003 